\title{
Interplay of environmental signals and progenitor diversity on fate specification of cortical GABAergic neurons
}

\author{
Juliana A. Brandão and Rodrigo N. Romcy-Pereira* \\ Brain Institute, Federal University of Rio Grande do Norte, Natal, Brazil
}

Cortical GABAergic interneurons constitute an extremely diverse population of cells organized in a well-defined topology of precisely interconnected cells. They play a crucial role regulating inhibitory-excitatory balance in brain circuits, gating sensory perception, and regulating spike timing to brain oscillations during distinct behaviors. Dysfunctions in the establishment of proper inhibitory circuits have been associated to several brain disorders such as autism, epilepsy, and schizophrenia. In the rodent adult cortex, inhibitory neurons are generated during the second gestational week from

OPEN ACCESS

Edited by:

Cecilia Hedin-Pereira

Federal University of Rio de Janeiro,

Brazil

Reviewed by:

Marco Canepari,

Institut National de la Santé et de la Recherche Médicale, France Xin Wang

Stanford University and Howard

Hughes Medical Institute, USA

*Correspondence:

Rodrigo N. Romcy-Pereira, Brain Institute, Federal University of Rio Grande do Norte, Avenida Nascimento de Castro, 2155 Natal,

RN 59056-450, Brazil rnrpereira@neuro.ufrn.br

Received: 11 December 2014 Accepted: 01 April 2015 Published: 28 April 2015

Citation:

Brandão JA and Romcy-Pereira RN (2015) Interplay of environmental signals and progenitor diversity on fate specification of cortical GABAergic neurons.

Front. Cell. Neurosci. 9:149. doi: 10.3389/fncel.2015.00149 distinct progenitor lineages located in restricted domains of the ventral telencephalon. However, only recently, studies have revealed some of the mechanisms generating the heterogeneity of neuronal subtypes and their modes of integration in brain networks. Here we will discuss some the events involved in the production of cortical GABAergic neuron diversity with focus on the interaction between intrinsically driven genetic programs and environmental signals during development.

Keywords: interneuron, cortical development, non-autonomous specification, inhibitory circuit, cell identity

\section{Introduction}

In mammals, the ability to produce their behavioral repertoire relies on a distributed network of neurons that coordinate the action of cortical, subcortical, and spinal cord circuits. From sensorimotor integration to executive functions, all depend on a precise spatio-temporal control of excitation and inhibition in local and long-range networks (Buzsáki, 2010). During brain development, cortical circuits are organized in a well-defined topology of interconnected excitatory and inhibitory neurons whose activities generate coherent behavioral outputs. Inhibitory neurons, in particular, play a crucial role fine-tuning neuronal firing to network oscillations and coordinating the emergence of task-relevant cell assemblies. In addition, GABAergic cells are important to promote the appropriate balance between excitation and inhibition required to prevent overexcitability and excitotoxicity (Somogyi and Klausberger, 2005). Disruption of this inhibitory control has been associated with several neurological disorders including autism, epilepsy, Down syndrome, Fragile X syndrome, X-linked lissencephaly with abnormal genitalia (XLAG), Rett syndrome and schizophrenia (Powell et al., 2003; Levitt et al., 2004; Guidotti et al., 2005; Ramamoorthi and Lin, 2011).

In the adult brain, cortical GABAergic interneurons constitute an extremely diverse population of cells comprising approximately $15-20 \%$ of all neurons depending on the cortical region. During 
the last 20 years, many studies have characterized and classified interneurons based on morphological and functional properties (DeFelipe, 1993; De Felipe et al., 1997; Kawaguchi and Kubota, 1997; Markram et al., 2004; Ascoli et al., 2008; Klausberger and Somogyi, 2008; Le Magueresse and Monyer, 2013). Although some of these properties are defining features, it is interesting to notice that at least some of them do not remain unchanged during lifetime. Studies have shown that some interneurons are able to change electrophysiological properties or the expression of endogenous molecular markers in response to environmental signals, resulting in functional plasticity in response to particular physiological demands (Steriade et al., 1998; Ascoli et al., 2008). Despite this complexity, accumulating evidence has suggested that none of these features alone can unambiguously define a homogeneous population of inhibitory neurons. In addition to the recent efforts to combine these elements and establish a common criterion to classify interneuron subgroups (Ascoli et al., 2008; DeFelipe et al., 2013), significant progress has been made characterizing the spatial distribution of subtype-specific GABAergic progenitors in the rodent embryo and its molecular determinants. Fate mapping experiments have revealed a group of segregated territories in the embryonic ventral telencephalon (subpallium) capable of generating all major populations of cortical GABAergic interneurons. In these proliferative niches, the patterned expression of transcription factors organize the early commitment of progenitors to specific neuronal fates (Parnavelas et al., 1991; Anderson et al., 1997; Sussel et al., 1999; Nery et al., 2002; Schuurmans and Guillemot, 2002; Xu et al., 2004; Wonders and Anderson, 2006; Flames et al., 2007; Guillemot, 2007; Miyoshi et al., 2010; Gelman et al., 2012). The combination of genetic fate mapping and cell transplantation studies have further revealed the contribution of environmental cues to the post-mitotic stages of interneuron specification thought to occur during migration, lamination, and establishment of synaptic contacts (Magrassi et al., 1998; Wichterle et al., 2001; Nery et al., 2002; Valcanis and Tan, 2003; Butt et al., 2005). In this review, therefore, we will discuss the mechanisms responsible for generating the diversity of GABAergic neurons during development and the interactions between environmental signals (extrinsic cues) and genetic programs (intrinsic factors) required to determine early (molecular) and late (molecular plus functional) cell identity, which will combine a proper laminar integration and functional maturation into specific inhibitory circuits.

\section{Diversity of GABAergic Cortical Interneurons}

Cortical GABAergic neurons are aspiny or sparsely spiny nonpyramidal cells that express the GABA-synthesizing enzyme, glutamic acid decarboxylase (GAD). They are found across all cortical layers and establish unique connections with excitatory and inhibitory cells in their vicinity. Some of these cells extend long horizontal or vertical axon collaterals within the cortex and have their cell bodies mostly dispersed into layers II-VI. In the adult brain, inhibitory synapses are precisely organized and contact specific sub-cellular compartments (soma, dendritic shafts, dendritic spines, axon initial segment, and pre-synaptic bouton) of particular neuronal types. This synaptic architecture, once formed, establishes the wiring pattern of local inhibitory circuits (Markram et al., 2004; Somogyi and Klausberger, 2005). Understanding cortical circuit function thus, requires a detailed appreciation of the diversity of GABAergic cells wired in the structure and their pattern of neuronal activity.

Some features can be used to define sub-types of GABAergic neurons: the somato-dendritic morphology, axonal arborization arrangement, post-synaptic sub-cellular target, biochemical identity, intrinsic electrophysiological properties, and modes of synaptic and structural plasticity (Gupta et al., 2000; Markram et al., 2004). As for their perisomatic and axonal arborization, cortical GABAergic interneurons are frequently identified as multipolar, bipolar or bitufted, chandelier, basket, or neurogliaform cells with axonal ramifications targeting distinct inhibitory domains on post-synaptic neurons. Basket cells for example show a perisomatic pattern of innervation establishing axosomatic and axo-dendritic symmetric contacts. In contrast, chandelier cells (or axo-axonic interneurons) contact exclusively the axon initial segment and multipolar Martinotti cells innervate distal dendrites of pyramidal neurons. Such variety of synaptic sites, aiming distinct sub-cellular compartments allows a precise inhibitory control over inputs from different cortical layers.

The molecular composition of GABAergic neurons is also diverse with the expression of calcium-binding proteins parvalbumin (PV), calretinin (CR) and calbindin (CB), and neuropeptides, such as somatostatin (SST), neuropeptide-Y (NPY), cholecystokinin (CCK) and vasoactive intestinal peptide (VIP; Houser et al., 1983; DeFelipe, 1993; Kawaguchi and Kubota, 1997; Gonchar, 2008). Other biochemical markers such as nitric oxide synthase (nNOS), reelin, serotonin receptor 3A (5HTR$3 \mathrm{~A}$ ) are also found in interneuron subpopulations such as ivy cells (neurogliaform), Cajal-Retzius neurons (transient embryonic layer I cells) and superficial layer neurons co-expressing VIP, respectively (Vucurovic et al., 2010; Jaglin et al., 2012). However recent findings have shown that three molecularly distinct non-overlapping populations of neurons can be sorted out according to the expression of PV, SST, and the ionotropic 5HTR-3A (Lee et al., 2010; Rudy et al., 2010). Interestingly, these neurons are born from spatially distinct domains of the embryo.

Interneurons also display a great diversity of intrinsic electrophysiological properties characterized by distinct firing modes in response to input stimuli or step-current injections. They can be classified according to their broad electrophysiological properties as non-accommodating ( $~ 50 \%)$, accommodating, (37\%) and stuttering cells $(\sim 13 \%)$ that can be further sub-grouped into classical spiking (regular fast-spiking and non-fast-spiking), bursting, and delayed onset response cells. Overall, it can be identified at least 14 individual functional sub-types of inhibitory cells (Gupta et al., 2000; Ascoli et al., 2008). Nevertheless, it is important to be cautious when considering this as static properties of a neuronal type. There still little information on inhibitory firing mode plasticity in animals in vivo. 
Parvalbumin- and somatostatin-expressing interneurons are the two most abundant classes of cortical interneurons with non-overlapping molecular identities and relatively large cell

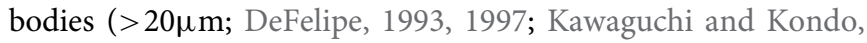
2002). GABAergic interneurons expressing PV make up $\sim 40 \%$ of all cortical interneurons of which basket and chandelier cells are typical members. These cells do not express SST, VIP, or CCK, but have some overlap labeling with CR and CB. They emit axonal collaterals to the soma, perisomatic dendrites and axons of post-synaptic targets. Functionally, these neurons show low-input resistance and fast-spiking dynamics of non-accommodating short-duration action potentials that impart a strong inhibitory control over their post-synaptic targets (Kawaguchi and Kubota, 1997; Gupta et al., 2000; Massi et al., 2012). PV-expressing inhibitory neurons represent an important population of cells involved in the modulation of critical period of auditory plasticity and implicated in psychiatric disorders, such as schizophrenia (Beasley and Reynolds, 1997; de Villers-Sidani et al., 2008). SST-expressing neurons, on the other hand, comprise $\sim 30 \%$ of the cortical inhibitory cells with soma preferentially distributed across deep cortical layers V-VI and axons branching into layer I where they exert inhibitory control over distal dendritic processes of pyramidal neurons. Martinotti cells, the most studied sub-population of SST-positive neurons so far, have multipolar somato-dendritic morphology and either regular adapting or intrinsically bursting activity. However, SST is also expressed in three other cell types: the murine X94 transgenic cell line located in layers IV$\mathrm{V}$ with projections to layer IV and either short-duration spikes or stuttering firing pattern (Ma et al., 2006) and, the cells named group 2-3 lines of short asymmetric axons that project to layers II/III. Yet they differ in their electrophysiological properties as one displays strongly adapting regular or stuttering firing modes, whereas the other have regular firing with narrower action potentials (McGarry et al., 2010). SST-positive neurons can also coexpress CR (21\%), NPY (7\%), nNOS, reelin, or CB in various proportions (Pesold et al., 1999; Xu et al., 2010b; Jaglin et al., 2012). The third class of cortical inhibitory neurons with nonoverlapping profiles with PV- and SST-positive cells expresses the ionotropic serotonin receptor 5HTR-3A. These cells comprise $\sim 20-30 \%$ of GABAergic neurons in the somatosensory cortex and represent the largest group of superficial neocortical interneurons (Morales and Bloom, 1997; Xu et al., 2010b). Despite the heterogeneity of co-labeling with CCK, NPY, and nNOS, two main subtypes of 5HTR-3A-expressing neurons can be distinguished: cells expressing VIP ( $\sim 40 \%$ of all $5 \mathrm{HTR}-3 \mathrm{~A}$ cells; located in layers II/III) and VIP-negative cells $(\sim 60 \%)$, most of them expressing reelin $(\sim 50 \%$; located in layer I). Lee et al. (2010) showed that more than $50 \%$ of VIP cells are of bitufted morphology and respond as irregular spiking neurons, while Miyoshi et al. (2010) described four distinct electrophysiological firing modes among VIP cells: burst non-adapting, delayed non-fast spiking, irregular spiking, and fast adapting cells. In contrast, most of VIP-negative GABAergic neurons are neurogliaform cells (reelin-positive) and fire accommodating delayed onset spikes in response to current steps (Lee et al., 2010).
Despite the great heterogeneity, GABAergic interneurons seem to select a group of neurons of the same class and establish synapses with homogenous temporal dynamics. In addition, a study using electrophysiological recordings obtained from pairs of different post-synaptic neurons following stimulation of the same pre-synaptic cell, showed that the same GABAergic axon can establish different types of synapses on distinct classes of target neurons (Gupta et al., 2000). Altogether, it is remarkable but still elusive, how such diversity is generated from a limited number of ventral telencephalic progenitors in the embryo.

\section{Diversity of Cortical Interneuron Progenitors}

During embryonic development, excitatory neurons are generated in the ventricular zone (VZ) of the dorsal telencephalon (pallium) and gradually invade the cortical plate by radial migration through specialized glial cells (Rakic, 2009). In the cortex, cell identity and laminar distribution are temporally organized by gene expression patterns and time of cell cycle exit (i.e., birthday), respectively (Angevine and Sidman, 1961; Rakic, 1988). In contrast, most of the brain's GABAergic interneurons derive from progenitors located in four different sub-regions of the subpallium: the medial (MGE), lateral (LGE) and caudal (CGE) ganglionic eminences and, the preoptic area (POA), which are defined by the expression of the homeodomain transcription factor $D l \times 1 / 2$ and the absence of Pax6 gene (Figure 1). Neural progenitors localized in the MGE, CGE, and POA give rise to virtually all cortical interneurons, whereas LGE progenitors produce GABAergic cell populations of olfactory bulb, amygdala, and striatum (Van Eden et al., 1989; Nery et al., 2002; Stuhmer et al., 2002; Xu et al., 2004; Flames et al., 2007; Gelman et al., 2011). It is important to mention that excitatory and inhibitory neurons are generated independently, in spatially segregated domains and do not share a common lineage. In the subpallium, regional identity is regulated by a combination of transcription factors with overlapping expression patterns, some of them showing cell fate restriction functions (Anderson et al., 1997; Sussel et al., 1999; Flames and Marin, 2005).

Medial ganglionic eminence progenitors are responsible for generating approximately $60 \%$ of all GABAergic neurons in the cortex. Around embryonic day 9 (E9), the action of the signaling molecule Shh (sonic hedgehog) on MGE cells induces the expression $N k \times 2.1$ and $L h \times 6$ transcription factors that orchestrates intracellular cascades required for the specification of SSTexpressing and PV-expressing interneurons (Butt et al., 2008; Du et al., 2008). Although $N k x 2.1$ and $\operatorname{Lhx6}$ specifically define the MGE neuroepithelium, $N k x 2.1$ is only briefly expressed in MGE progenitors. In a cascade of events, $L h x 6$, a direct target of $N k \times 2.1$, is required for the acquisition of molecular identity and laminar positioning (Sussel et al., 1999; Liodis et al., 2007) and Sox6 and Satb1 seem to act as downstream effectors of Lhx6 (Azim et al., 2009; Batista-Brito et al., 2009; Close et al., 2012). Analysis of $\operatorname{Lh} x 6$ mutant mice has shown that even though Lh $x 6^{-/-}$progenitors are still able to migrate to the pallium, most derived interneurons lack PV and SST expression and show abnormal laminar integration (Zhao et al., 2008). Interestingly, $\mathrm{Xu}$ et al. (2010a) have recently demonstrated that high levels 


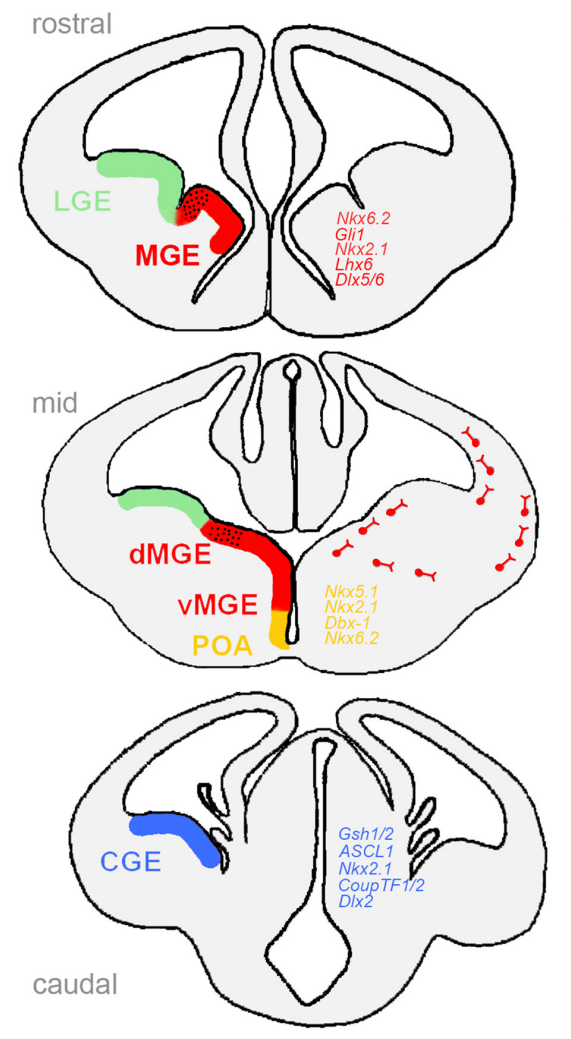

FIGURE 1| Ventral telencephalic germinative zones of cortical GABAergic neurons in the rodent embryonic brain. The medial ganglionic eminence (dorsal, dMGE; ventral, VMGE), caudal ganglionic eminence (CGE), and the preoptic area (POA) are responsible for generating virtually all cortical interneurons. In each region, neural progenitor classes are defined by a combinatorial action of transcription factors (Left) that restrict cells to neurochemically defined fates (Right). Distinct classes of inhibitory cells display characteristic morphology, laminar distribution and electrophysiological properties.

\section{Embryo E13.5}

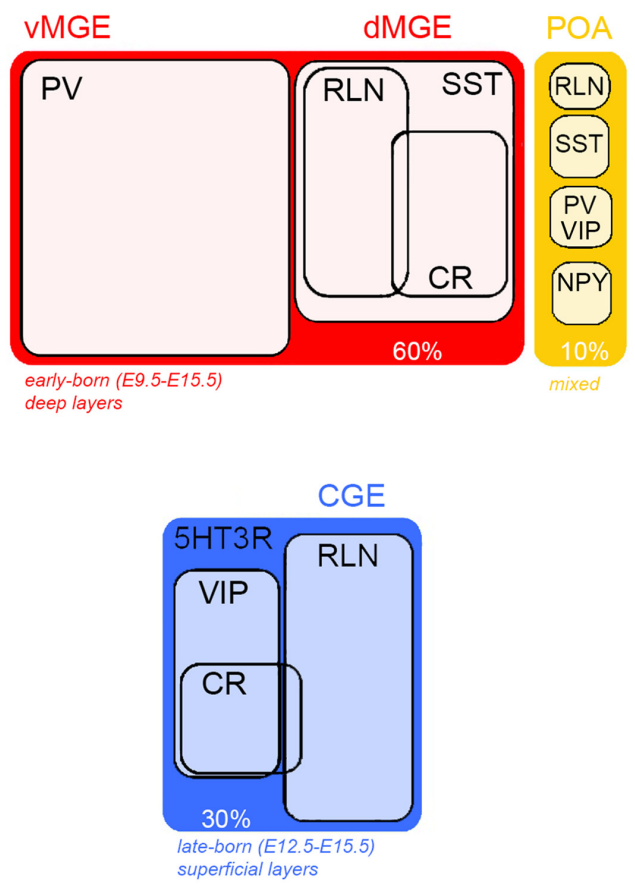

5HT3R, serotonin receptor type 3; CR, calretinin; NPY, neuropeptide Y; PV, parvalbumin; RLN, reelin; SST, somatostatin; VIP, vasointestinal active peptide. Genes: ASCL1, achaete-scute family $b H L H$ transcription factor 1 (Mash1, mammalian homologue); Dbx-1, developing brain homeobox 1; CoupTF1/2, chicken ovalbumin upstream promoter transcription factor; $D / x(D / \times 1 / 2 / 5 / 6)$, distal-less homeobox; Gli1, Gli family zinc finger 1; Gsh1/2, GS homeobox 2; Lhx6, LIM homeobox 6; Nkx (Nkx2.1, Nkx5.1, Nkx6.2), NK homeobox family. of Shh in dorsal MGE compared to ventral MGE is responsible for a dorso-ventral patterning of progenitors in this region, suggesting an important role for soluble factors in early fate determination in the subpallium. As a result, two different territories in the MGE can be identified. The dorsal division (dMGE) is enriched in Nkx6.2 and Gli1 genes, and preferentially gives rise to SST-expressing interneurons comprising about $65 \%$ of all MGEderived neurons, including Martinotti cells that co-express CR, NPY-expressing cells and all nNOS-positive neurons. The ventral division (vMGE), in contrast, is enriched in Dlx5/6 and Lhx6 genes and generates most of the PV-expressing interneurons of the cortex $(\sim 35 \%)$ that includes large basket and chandelier cells (Fogarty et al., 2007; Jaglin et al., 2012).

These two lineages of neurons are born around E12.5E16.5 (peak E14.5) and migrate tangentially toward the pallium through the subventricular zone (SVZ) and marginal zones (MZ) to be subsequently incorporated into the cortical plate (Butt et al., 2005; Miyoshi et al., 2007). They follow a temporal-positioning code according to their birthdate showing an inside-out neurogenic gradient of lamination. Although the final distribution of MGE-derived neurons encompasses preferentially deep cortical layers (layers IV-VI), some neurons have relatively restricted laminar patterns as seen for chandelier cells that prefer layers II and IV (Taniguchi et al., 2013).

The other major contributing region for the generation of cortical GABAergic neurons is the CGE that can be considered the caudal extension of MGE and LGE in the ventral telencephalon. The CGE is responsible for producing about $30 \%$ of all adult cortical interneurons, of which virtually all express the serotonin receptor subtype 3a (5HT3aR; Rudy et al., 2010; Vucurovic et al., 2010). Interneurons from the CGE have bipolar or doublebouquet morphology and display electrophysiological characteristics of irregular firing or fast-adapting cells. Electrophysiological studies have grouped these cells in more than six different subtypes according to their firing patterns and morphology (Lee et al., 2010; Miyoshi et al., 2010). Forty percent of these neurons co-express VIP (some also expressing CR, but negative for SST), whereas about $80 \%$ of the remaining VIP-negative cells express the extracellular signaling protein reelin (Nery et al., 2002; Lee et al., 2010; Miyoshi et al., 2010; Jaglin et al., 2012). Overall, 
CR-positive/SST-negative cells, as well as the great majority of VIP-, CCK-, and reelin-expressing GABAergic interneurons are derived from $5 \mathrm{HT} 3 \mathrm{aR}$-expressing CGE progenitors.

These progenitors can also be identified by an abundant expression of the transcription factors CoupTF1/2, Gsh1/2 (Sousa et al., 2009; Lee et al., 2010). Gsh2 is enriched in the CGE and controls the expression of Mash1, ASCL1, Dlx2 required for CGE patterning. In fact, there is evidence suggesting an internal regionalization of the CGE, in which the dorsal region expresses Gsh 2 and induces the proneural gene Mash1 and Delta (a Notch ligand), leading to repression of progenitor differentiation, whereas the ventral CGE is enriched in NKx2.1. The consequences of this molecular regionalization are still not clear.

Caudal ganglionic eminence-derived neurons are late-born cells with peak production around E16.5, migrating tangentially toward the pallium through the SVZ and MZ (Cavanagh and Parnavelas, 1989). They do not follow a temporal-positioning code, but instead get distributed homogeneously across superficial cortical layers (layers I-III), with $\sim 40 \%$ occupancy in layers II and III. An exception to this rule is the outside-in neurogenic gradient observed in CR-positive interneurons (Lee et al., 2010).

In the ventro-medial region of the subpallium, the POA generates the remaining $10 \%$ of cortical interneurons, including multiple classes of GABAergic cells. They consist of a small fraction of PV-, SST-, NPY-, and reelin-expressing cells, all derived from POA progenitors with transcriptional programs distinct from those of the MGE and CGE (Gelman et al., 2011). Interestingly, mice lacking Lhx6 expression (a specific marker of MGE progenitors) are almost completely depleted of PV-positive and SST-positive neurons, except for some scattered cells possibly derived from POA, seen in deep layers of the cortex (Liodis et al., 2007; Zhao et al., 2008). Fate determination in the POA seems to be orchestrated by the action of several genes under the instruction of Shh and Nkx2.1. Using genetic fate-mapping and transcription factor expression analysis, it has been shown that POA contains at least two progenitor domains defined by the non-overlapping cellular expression of $D b x-1, N k x 6.2$ (ventrally), and Nkx5.1 (dorsally; Flames et al., 2007; Gelman et al., 2011). About $40 \%$ of the POA-derived interneurons are generated by Dbx-1-expressing progenitors, most of them born on E12.5, distributed across layers V and VI of the cortex and expressing PV, SST, and reelin. On the other hand, Nkx5.1-derived interneurons are mostly found in superficial layers of the cortex and constitute a rather homogeneous population of rapidly adapting interneurons, many of which express NPY. However, it is still not clear so far how such diversity of interneuron subtypes arise from the Dbx-1-expressing lineage (Gelman et al., 2009).

\section{Role of Environmental Factors on Cortical Interneuron Differentiation}

Cortical interneurons acquire much of their adult identity by the time of birth (i.e., after the last cell division), due to early fate restriction imposed by genetic programs (Xu et al., 2005; Flames et al., 2007; Fogarty et al., 2007; Miyoshi et al., 2007; Butt et al., 2008; Azim et al., 2009; Batista-Brito et al., 2009; Neves et al., 2013; Kessaris et al., 2014; Vogt et al., 2014). However, many of the characteristics used to define interneurons are not evident until late postnatal ages and even adulthood. Therefore, it is reasonable to inquire what are the post-mitotic events necessary to assure proper development of cortical inhibitory circuits. Recent findings have given support to the idea that interactions with environmental signals starting from early post-mitotic stages and all way along their migratory path to final destination are essential for the full expression of mature features (Valcanis and Tan, 2003; Rouaux and Arlotta, 2010; De Marco García et al., 2011; Lodato et al., 2011; Close et al., 2012; Denaxa et al., 2012; Spitzer, 2012). Some of the features that undergo protracted specification include cortical area assignment, laminar positioning, neurochemical identity, dendritic arbor topology, and post-synaptic cell targeting.

Molecular signals in the environment as well as activitydependent membrane currents seem to be necessary cues to guide the execution and fulfillment of genetic programs set in motion during early stages of interneuron development (Figure 2). For instance, evidences show that manipulations of these environmental cues surrounding neural progenitors affect the neurochemical identity of mature interneurons. Resident pyramidal neurons, electrical activity, and local molecular codes are thought to instruct intrinsic programs and promote neuronal migration, layer positioning, cell target selection, and the precise apposition of subcellular synaptic contacts. Here we will discuss some of these studies.

\section{Pyramidal Cells Controls Interneuron Laminar Identity}

Laminar positioning of interneurons in the cortex depends both upon their place of origin and date of birth (Miyoshi and Fishell, 2011), however, evidence points to a more interactive and epigenetic perspective on interneuron laminar specification. Valcanis and Tan (2003) have shown that early- and late-born interneuron progenitors have their laminar fate re-specified by extrinsic signals of the new environment. Using transplantation experiments, the authors show that donor progenitor cells can acquire the host laminar distribution once they undergo a last round of cell division in the host tissue (Valcanis and Tan, 2003).

It was also observed that the interaction of migrating interneurons with resident pyramidal cells control the laminar identity of GABAergic cells. Pla et al. (2006) showed that disrupting the normal layering of pyramidal neurons, in Dab1-/- mice deficient in reelin signaling, modifies the laminar allocation of MGEderived cells. Wild-type interneurons transplanted into the cortex of Dab1 mutants closely matched the layer distribution of host pyramidal neurons, whereas Dab1-/- interneurons transplanted in wild-type cortex were able to generate normal interneuron positioning, as if guided by well-positioned local pyramidal cells (Pla et al., 2006). Collectively, these findings support a reelinindependent mechanism where pyramidal neurons are required for proper interneuron integration. The authors also noticed that more than $20 \%$ of early born (E12) and $\sim 60 \%$ of late born (E15) wild-type interneurons change their laminar fate upon transplantation into older (E15) developing wild-type cortex.

In agreement with the previous results, Lodato et al. (2011) have recently shown that SST- and PV-expressing interneurons are specifically reduced in layer 5 of Fezf2-depleted mice, in which 
A PYRAMIDAL CELL GUIDANCE

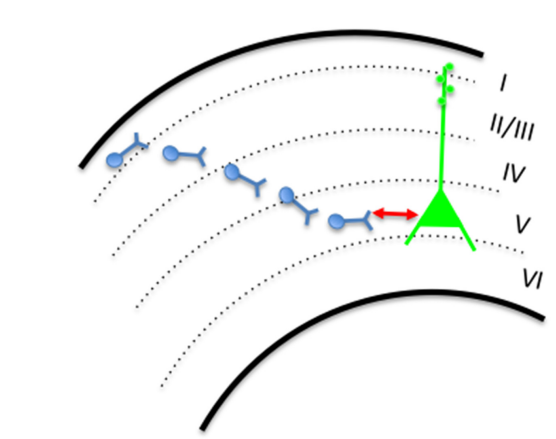

B ACTIVITY-DEPENDENT MECHANISM

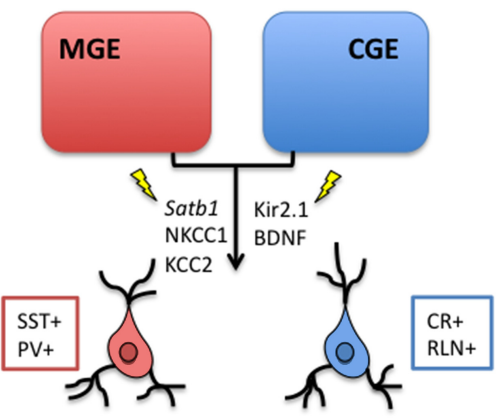

C SECRETED SOLUBLE FACTORS

FIGURE 2 | Mechanisms regulating post-mitotic plasticity and late-fate specification of cortical GABAergic interneurons. (A) Local pyramidal cells modulate laminar positioning of inhibitory neurons in the cortex (Pla et al., 2006; Lodato et al., 2011). (B) Electrical activity (GABA-mediated membrane depolarization, $\mathrm{Ca}^{2+}$ transients) can regulate late acquisition of molecular identity in MGE- and CGE-derived interneurons (Berghuis et al., 2004: Borodinsky et al., 2004; De Marco García et al., 2011; Close et al., 2012; Denaxa et al., 2012). (C) Soluble secreted factors can alter the balance of interneuron subtypes through shift in neurochemical identity (Xu et al., 2005; Lopez-Bendito et al., 2008; Cambray et al., 2012; Vogt et al., 2014). subcerebral pyramidal projection neurons are absent from the cortex. The lack of glutamatergic projection neurons in layer 5 of these animals creates an abnormal distribution of PV and SST neurons, which suggests that these neurons control the laminar positioning of inhibitory migrating cells in the cortex (Lodato et al., 2011).

\section{Electrical Activity Instructs Phenotypic, Laminar and Synaptic Identity}

Another environmental signal that regulates late fate specification of cortical interneurons is electrical activity. In CGEderived neurons, normal excitability is required for proper integration into their final destination. Using in utero electroporation with the inward rectifying potassium channel Kir2.1 directed to CGE-derived neurons, De Marco García et al. (2011) showed that depolarization was necessary for the full phenotypic development of CR- and reelin-expressing cells. They observed morphological defects in the axonal arbor and dendritic tree of both neuron subtypes or only in reelin-expressing cells under hyperpolarization, respectively. Although tangential migration was unchanged, it was detected a shift in laminar positioning when potassium currents were induced after post-natal day 5 (P5) in CR+ and Re+ CGE-derived neurons. These results indicate that genetic programs initiated at progenitor stage can be modulated by electrical activity during development.
Furthermore, Berghuis et al. (2004) have shown that neuronal depolarization of PV-expressing neurons maintained in culture enhances the BDNF differentiating effects. While BDNF promotes dendritic branching, somatic differentiation, strengthening of synaptic connections, and frequency modulation of action potentials, the addition of $\mathrm{KCl}$ (i.e., depolarization) was required for the establishment of reciprocal inhibitory synapses and significantly accelerated the formation of synaptic contacts (Berghuis et al., 2004). Consistent with this idea, GABA and glutamate play an important role in post-mitotic cortical neuronal motility as it modulates intracellular calcium $\left(\mathrm{Ca}^{2+}\right)$ transients through GABAA and NMDA/AMPA receptor-mediated depolarization. In contrast, hyperpolarizing GABA decreases the magnitude of $\mathrm{Ca}^{2+}$ transients inhibiting motility of migrating cells. After reaching the cortex, migrating interneurons upregulate the potassium/chloride $\left(\mathrm{K}^{+} / \mathrm{Cl}^{-}\right)$exchanger $\mathrm{KCC} 2$, which determines the developmental switch from depolarizing to hyperpolarizing action of GABA (Ben-Ari, 2002). KCC2 expression thus can act as a switch to induce a voltage-sensitive, $\mathrm{Ca}^{2+}$-mediated reduction of interneuron motility and function as a migration stop signal (Bortone and Polleux, 2009). Indeed, migrating cortical interneurons may sense and integrate the ambient, local extracellular levels of GABA and glutamate as a way to determine when to stop migration. In the ganglionic eminence, progenitors are the main source of GABA and MGE-derived interneurons begin to express KCC2 a few hours 
after reaching the cortex (Hevner et al., 2004; Inamura et al., 2012)

$\mathrm{Ca}^{2+}$ spikes also modulate the neurochemical specification of neural progenitors between GABA/glycine and glutamate/acetylcholine in the dorso-ventral axis of neural tube (Borodinsky et al., 2004). Cells exhibiting high frequency of $\mathrm{Ca}^{2+}$ spikes express GABA and glycine, whereas cells with a low spike frequency take the glutamatergic and cholinergic phenotype. In the spinal cord, $\mathrm{Ca}^{2+}$-mediated signaling contributes to proliferation, migration, axon pathfinding, dendritic growth, and specification of neurotransmitter subtype (Rosenberg and Spitzer, 2011).

Another particular example concerns the chromatin organizer and transcription factor Satb1 (special AT-rich binding protein) that is specifically expressed in mature interneurons located in the cortical plate. Two recent studies have demonstrated that Satb1 is a key molecule acting downstream Lhx6 to control maturation and late differentiation of cortical interneurons in an activity-dependent manner. Ablation of Satb1 in mice promotes a dramatic decrease of SST mRNA and protein expression in postnatal cortical interneurons, but no effect on the expression in migrating precursors (Close et al., 2012; Denaxa et al., 2012). Close et al. (2012) showed that embryonic deletion of Satb1 in MGE-derived interneurons disrupts the migration and synaptic integration of both PV- and SST-expressing neurons into nascent cortical circuits with special effect on the differentiation of SST-expressing cells. Interestingly, Satb1 is regulated by neuronal activity, which is required for the establishment of mature patterns of inhibition onto cortical pyramidal cells (Close et al., 2012). The second study showed that treatment of dorsal telencephalon cell cultures with $\mathrm{KCl}$ induced the expression of Satb1 and c-FOS in cortical GABAergic neurons within $24 \mathrm{~h}$. In addition, Satb1 was shown to be required for the induction of the neurochemical phenotype typical of mature SST-expressing GABAergic neurons and was regulated by neuronal excitability involving $\mathrm{Ca}^{2+}$ influx and GABA receptor activation. Using misexpression experiments in the MGE of E14.5 embryos, the authors observed that Satb1 could further affect neuronal activity by modulating the expression of NKCC1 $\left(\mathrm{a} \mathrm{Na}^{+}-\mathrm{K}^{+}-\mathrm{Cl}^{-}\right.$ co-transporter abundantly expressed in immature GABAergic interneurons) and KCC2 (Denaxa et al., 2012). KCC2 expression by its turn could set off the termination of cortical interneuron migration in a voltage-sensitive and calcium-dependent manner.

\section{Soluble Factors Shape Cell Identity}

Another possible mechanism of post-mitotic plasticity is the interaction of migrating interneurons with soluble molecules in the environment. In an early study, Iacovitti et al. (1987) observed that post-mitotic neurons obtained from E13 embryonic cortical cultures, grown for 1 day, expressed a catecholaminergic phenotype different from what was observed in vivo in the cortex. The authors then suggested that external factors might have interfered with neurotransmitter specification in vitro that did not occur in vivo (Iacovitti et al., 1987).

Other studies further revealed that environmental signals could in fact regulate neurochemical identity of GABAergic neurons. In a first report, dissociated cortical interneurons prepared at birth failed to express VIP at P2, whereas interneurons from neonatal slices of same age did express the neuropeptide (Gotz and Bolz, 1994). Following that Gulacsi and Lillien (2003) showed that dorsal telencephalic progenitors co-cultured with ventral progenitors generated more GABAergic neurons than in single culture. This effect was reduced or enhanced by the addition of cyclopamine, an antagonist of Shh or exogenous Shh, respectively (Gulacsi and Lillien, 2003). New evidence to Shh involvement came from Xu et al. (2005) showing that dissociated interneuron cells treated with cyclopamine expressed reduced PV and SST phenotypes. Moreover, using a conditional mutant mice NestinCre:Shh(flox/flox), in which Shh signaling is deficient, they found altered MGE patterning and reduced number of NPY, PV, and SST neurons in the somatosensory cortex (Xu et al., 2005). In fact, Shh seems to maintain the identity of cortical interneuron progenitors in the ventral telencephalon through a continuous provision of positional information by regulating $N k \times 2.1$ expression. In this manner, it plays a critical role in determining the relative composition of cortical excitatory and inhibitory neurons.

It is known that post-mitotic GABAergic neurons are guided toward the cortex along two main migratory streams (MZ and SVZ) by the interaction with soluble chemoattractants and chemorepellents dispersed in the pathway. Among them, Cxcl12 (and its receptors, Cxcr4, Cxcr7), a potent chemoattractant to MGE-derived interneurons is required for normal positioning of interneurons in the cortex ( $\mathrm{Li}$ et al., 2008; Lopez-Bendito et al., 2008). Recently, it was reported that Arx and Cxcr7 (Lhx6-target genes) could rescue the cell identity and laminar phenotype of Lhx6 $6^{-/-}$MGE cells. In Lhx6 mutant mice, PV and SST-expressing neurons are specifically reduced and some cells acquire CGE-like fate. $L h x 6 / 8$ are known to be necessary for Shh expression in MGE and to determine cell fate of PV- and SST-neurons. Besides, $L h x 6 / 8$ are also required for repressing $D l x 1$ in the pallium, preventing GABAergic fate of local progenitors (Flandin et al., 2011; Vogt et al., 2014).

Another example of a soluble molecule with putative fate specification role is activin. It is a member of the TGF-3 family of neurotrophic factors, produced in LGE/CGE and shown to induce telencephalic precursors to adopt LGE/CGE cell fate. Through the specific induction of Gsh2 expression and inhibition of Shh and Nkx2.1, it promotes the acquisition of CGE fate and CR phenotype (Cambray et al., 2012). In fact, neurotrophins were already shown to regulate the relative number of GABAergic and cholinergic neurons in the rodent basal forebrain through their action on p75 receptor during development (Lin et al., 2007). Moreover, some studies show that serotonin depletion transiently delays the incorporation of CGE-derived interneurons into the cortical plate and alters the maturation of CR- and CCK-expressing interneurons in the somatosensory cortex. Such depletion also decreases reelin secretion by CajaRetzius cells leading to dendritic hyper-complexity in pyramidal neurons (Vitalis et al., 2007, 2013).

More recently, Díaz-Alonso et al. (2012), using in vitro and in vivo experiments, observed that embryonic endocannabinoids 
(eCB) regulate the intrinsic program for layer fate specification of cortical pyramidal neurons through CB1 receptors. The authors showed that CB1 signaling controls the appropriate balance of neuronal differentiation in deep cortical layers by inhibiting Satb2 and promoting Ctip2 expression following extracellular levels of eCB. Cortico-spinal and subcerebral projection neurons were particularly affected. Interestingly, CB1 receptor inactivation decreased the expression of deep-layer markers Fezf2 and Ctip2 in pyramidal cells, but did not affect Ctip2 expression in GABAergic neurons (Díaz-Alonso et al., 2012). However, considering that laminar identity of cortical GABAergic interneurons can be regulated by resident glutamatergic cells, these findings also suggest that $\mathrm{eCB}$ might indirectly govern the laminar allocation of interneurons.

Another indirect effect on the final organization of cortical interneuron circuits may be exerted by the neurotrophin 3 (Nt3). Through the repressor Sip1, Nt3 acts as a feedback signal between post-mitotic and progenitors neurons. It is able to switch the cell fate of apical progenitors and promote overproduction of superficial layer cortical neurons in the developing mouse. Changing the balance of superficial and deep projection neurons, Nt3 might affect interneurons layer positioning. Sip1 could

\section{References}

Anderson, S. A., Eisenstat, D. D., Shi, L., and Rubenstein, J. L. (1997). Interneuron migration from basal forebrain to neocortex: dependence on Dlx genes. Science $278,474-476$.

Angevine, J. B. Jr., and Sidman, R. L. (1961). Autoradiographic study of cell migration during histogenesis of cerebral cortex in the mouse. Nature 192, 766-768.

Ascoli, G. A., Alonso-Nanclares, L., Anderson, S. A., Barrionuevo, G., BenavidesPiccione, R., Burkhalter, A., et al. (2008). Petilla terminology: nomenclature of features of GABAergic interneurons of the cerebral cortex. Nat. Rev. Neurosci. 9, 557-568. doi: 10.1038/nrn2402

Azim, E., Jabaudon, D., Fame, R. M., and Macklis, J. D. (2009). SOX6 controls dorsal progenitor identity and interneuron diversity during neocortical development. Nat. Neurosci. 12, 1238-1247. doi: 10.1038/ nn. 2387

Batista-Brito, R., Rossignol, E., Hjerling-Leffler, J., Denaxa, M., Wegner, M., Lefebvre, V., et al. (2009). The cell-intrinsic requirement of Sox6 for cortical interneuron development. Neuron 63, 466-481. doi: 10.1016/j.neuron.2009.08.005

Beasley, C. L., and Reynolds, G. P. (1997). Parvalbumin-immunoreactive neurons are reduced in the prefrontal cortex of schizophrenics. Schizophr. Res. 24, 349-355.

Ben-Ari, Y. (2002). Excitatory actions of gaba during development: the nature of the nurture. Nat. Rev. Neurosci. 3, 728-739. doi: 10.1038/nrn920

Berghuis, P., Dobszay, M. B., Sousa, K. M., Schulte, G., Mager, P. P., Hartig, W., et al. (2004). Brain-derived neurotrophic factor controls functional differentiation and microcircuit formation of selectively isolated fast-spiking GABAergic interneurons. Eur. J. Neurosci. 20, 1290-1306. doi: 10.1111/j.14609568.2004.03561.x

Borodinsky, L. N., Root, C. M., Cronin, J. A., Sann, S. B., Gu, X., and Spitzer, N. C. (2004). Activity-dependent homeostatic specification of transmitter expression in embryonic neurons. Nature 429, 523-530. doi: 10.1038/ nature 02518

Bortone, D., and Polleux, F. (2009). KCC2 expression promotes the termination of cortical interneuron migration in a voltage-sensitive calcium-dependent manner. Neuron 62, 53-71. doi: 10.1016/j.neuron.2009.01.034

Butt, S. J. B., Fuccillo, M., Nery, S., Noctor, S., Kriegstein, A., Corbin, J. G., et al. (2005). The temporal and spatial origins of cortical interneurons predict also switch progenitors fate from neurogenesis to gliogenesis (Seuntjens et al., 2009; Parthasarathy et al., 2014).

Finally, the number of specific interneuronal subtypes in the cortex and their final fate was shown to be critically determined by the regulation of cell cycle in neuronal progenitors. Actually, it was observed that cyclin D2 delays cell cycle exit of MGE progenitors and its deletion leads to a prominent reduction in PV-positive cells in the mature rodent cortex along with microcephalic phenotype (Glickstein et al., 2007). Interestingly, cyclin D2 expression seems to be regulated by $\mathrm{Nt} 3$ and Shh (Lukaszewicz et al., 2002; Komada et al., 2013).

\section{Conclusion}

Cell fate specification of cortical interneurons seems to require the interplay of both intrinsic and extrinsic molecular signals. However, the distinct aspects of such delicate control just began to be unveiled. Time of signaling, cell-type targeting, magnitude of phenotypic effects, and the particular molecular mechanisms involved are still unknown. Future experiments should bring some light on these open questions.

their physiological subtype. Neuron 48, 591-604. doi: 10.1016/j.neuron.2005. 09.034

Butt, S. J. B., Sousa, V. H., Fuccillo, M. V., Hjerling-Leffler, J., Miyoshi, G., Kimura, S., et al. (2008). The requirement of Nkx2-1 in the temporal specification of cortical interneuron subtypes. Neuron 59, 722-732. doi: 10.1016/j.neuron.2008.07.031

Buzsáki, G. (2010). Neural syntax: cell assemblies, synapsembles, and readers. Neuron 68, 362-385. doi: 10.1016/j.neuron.2010.09.023

Cambray, S., Arber, C., Little, G., Dougalis, A. G., De Paola, V., Ungless, M. A., et al. (2012). Activin induces cortical interneuron identity and differentiation in embryonic stem cell-derived telencephalic neural precursors. Nat. Commun. 3, 841. doi: $10.1038 /$ ncomms 1817

Cavanagh, M. E., and Parnavelas, J. G. (1989). Development of vasoactiveintestinal-polypeptide-immunoreactive neurons in the rat occipital cortex: a combined immunohistochemical-autoradiographic study. J. Comp. Neurol. 284, 637-645. doi: 10.1002/cne.902840410

Close, J., Xu, H., De Marco Garcia, N., Batista-Brito, R., Rossignol, E., Rudy, B., et al. (2012). Satb1 is an activity-modulated transcription factor required for the terminal differentiation and connectivity of medial ganglionic eminence-derived cortical interneurons. J. Neurosci. 32, 17690-17705. doi: 10.1523/JNEUROSCI.3583-12.2012

DeFelipe, J. (1993). Neocortical neuronal diversity: chemical heterogeneity revealed by colocalization studies of classic neurotransmitters, neuropeptides, calcium-binding proteins, and cell surface molecules. Cereb. Cortex 3, 273-289.

DeFelipe, J. (1997). Types of neurons, synaptic connections and chemical characteristics of cells immunoreactive for calbindin-D28K, parvalbumin and calretinin in the neocortex. J. Chem. Neuroanat. 14, 1-19.

DeFelipe, J., López-Cruz, P. L., Benavides-Piccione, R., Bielza, C., Larrañaga, P., Anderson, S., et al. (2013). New insights into the classification and nomenclature of cortical GABAergic interneurons. Nat. Rev. Neurosci. 14, 202-216. doi: $10.1038 / \mathrm{nrn} 3444$

De Felipe, J., Marco, P., Fairén, A., and Jones, E. G. (1997). Inhibitory synaptogenesis in mouse somatosensory cortex. Cereb. Cortex 7, 619-634.

De Marco García, N. V., Karayannis, T., and Fishell, G. (2011). Neuronal activity is required for the development of specific cortical interneuron subtypes. Nature 472, 351-355. doi: 10.1038/nature09865

de Villers-Sidani, E., Simpson, K. L., Lu, Y. F., Lin, R. C., and Merzenich, M. M. (2008). Manipulating critical period closure across different sectors 
of the primary auditory cortex. Nat. Neurosci. 11, 957-965. doi: 10.1038/ nn. 2144

Denaxa, M., Kalaitzidou, M., Garefalaki, A., Achimastou, A., Lasrado, R., Maes, T., et al. (2012). Maturation-promoting activity of SATB1 in MGE-derived cortical interneurons. Cell Rep. 2, 1351-1362. doi: 10.1016/j.celrep.2012.10.003

Díaz-Alonso, J., Aguado, T., Wu, C.-S., Palazuelos, J., Hofmann, C., Garcez, P., et al. (2012). The CB(1) cannabinoid receptor drives corticospinal motor neuron differentiation through the Ctip2/Satb2 transcriptional regulation axis. J. Neurosci. 32, 16651-16665. doi: 10.1523/JNEUROSCI.0681-12.2012

Du, T., Xu, Q., Ocbina, P. J., and Anderson, S. A. (2008). NKX2.1 specifies cortical interneuron fate by activating Lhx6. Development 135, 1559-1567. doi: 10.1242/dev.015123

Flames, N., and Marin, O. (2005). Developmental mechanisms underlying the generation of cortical interneuron diversity. Neuron 46, 377-381. doi: 10.1016/j.neuron.2005.04.020

Flames, N., Pla, R., Gelman, D. M., Rubenstein, J. L. R., Puelles, L., and Marín, O. (2007). Delineation of multiple subpallial progenitor domains by the combinatorial expression of transcriptional codes. J. Neurosci. 27, 9682-9695. doi: 10.1523/JNEUROSCI.2750-07.2007

Flandin, P., Zhao, Y., Vogt, D., Jeong, J., Long, J., Potter, G., et al. (2011). Lhx6 and Lhx8 coordinately induce neuronal expression of Shh that controls the generation of interneuron progenitors. Neuron 70, 939-950. doi: 10.1016/j.neuron.2011.04.020

Fogarty, M., Grist, M., Gelman, D., Marín, O., Pachnis, V., and Kessaris, N. (2007). Spatial genetic patterning of the embryonic neuroepithelium generates GABAergic interneuron diversity in the adult cortex. J. Neurosci. 27, 10935-10946. doi: 10.1523/JNEUROSCI.1629-07.2007

Gelman, D., Griveau, A., Dehorter, N., Teissier, A., Varela, C., Pla, R., et al. (2011). A wide diversity of cortical gabaergic interneurons derives from the embryonic preoptic area. J. Neurosci. 31, 16570-16580. doi: 10.1523/jneurosci.406811.2011

Gelman, D. M., Marín, O., and Rubenstein, J. L. R. (2012). “The generation of cortical interneurons," in Jesper's Basic Mechanisms of the Epilepsies, 4th Edn, eds J. Noebels, M. Avoli, M. Rogawski, R. Olsen, and A. Delgado-Escueta (New York: Oxford University Press).

Gelman, D. M., Martini, F. J., Nobrega-Pereira, S., Pierani, A., Kessaris, N., and Marin, O. (2009). The embryonic preoptic area is a novel source of cortical GABAergic interneurons. J. Neurosci. 29, 9380-9389. doi: 10.1523/JNEUROSCI.0604-09.2009

Glickstein, S. B., Moore, H., Slowinska, B., Racchumi, J., Suh, M., Chuhma, N., et al. (2007). Selective cortical interneuron and GABA deficits in cyclin D2-null mice. Development 134, 4083-4093. doi: 10.1242/dev.008524

Gonchar, Y. (2008). Multiple distinct subtypes of GABAergic neurons in mouse visual cortex identified by triple immunostaining. Front. Neuroan. 1:3. doi: 10.3389/neuro.05.003.2007

Gotz, M., and Bolz, J. (1994). Differentiation of transmitter phenotypes in rat cerebral cortex. Eur. J. Neurosci. 6, 18-32.

Guidotti, A., Auta, J., Davis, J. M., Dong, E., Grayson, D. R., Veldic, M., et al. (2005). GABAergic dysfunction in schizophrenia: new treatment strategies on the horizon. Psychopharmacology 180, 191-205. doi: 10.1007/s00213-005-2212-8

Guillemot, F. (2007). Cell fate specification in the mammalian telencephalon. Progr. Neurobiol. 83, 37-52. doi: 10.1016/j.pneurobio.2007.02.009

Gulacsi, A., and Lillien, L. (2003). Sonic hedgehog and bone morphogenetic protein regulate interneuron development from dorsal telencephalic progenitors in vitro. J. Neurosci. 23, 9862-9872.

Gupta, A., Wang, Y., and Markram, H. (2000). Organizing principles for a diversity of GABAergic interneurons and synapses in the neocortex. Science 287, 273-278.

Hevner, R. F., Daza, R. A., Englund, C., Kohtz, J., and Fink, A. (2004). Postnatal shifts of interneuron position in the neocortex of normal and reeler mice: evidence for inward radial migration. Neuroscience 124, 605-618. doi: 10.1016/j.neuroscience.2003.11.033

Houser, C. R., Hendry, S. H., Jones, E. G., and Vaughn, J. E. (1983). Morphological diversity of immunocytochemically identified GABA neurons in the monkey sensory-motor cortex. J. Neurocytol. 12, 617-638.

Iacovitti, L., Lee, J., Joh, T. H., and Reis, D. J. (1987). Expression of tyrosine hydroxylase in neurons of cultured cerebral cortex: evidence for phenotypic plasticity in neurons of the CNS. J. Neurosci. 7, 1264-1270.
Inamura, N., Kimura, T., Tada, S., Kurahashi, T., Yanagida, M., Yanagawa, Y., et al. (2012). Intrinsic and extrinsic mechanisms control the termination of cortical interneuron migration. J. Neurosci. 32, 6032-6042. doi: 10.1523/JNEUROSCI.3446-11.2012

Jaglin, X. H., Hjerling-Leffler, J., Fishell, G., and Batista-Brito, R. (2012). The origin of neocortical nitric oxide synthase-expressing inhibitory neurons. Front. Neural Cir. 6:44. doi: 10.3389/fncir.2012.00044

Kawaguchi, Y., and Kondo, S. (2002). Parvalbumin, somatostatin and cholecystokinin as chemical markers for specific GABAergic interneuron types in the rat frontal cortex. J. Neurocytol. 31, 277-287.

Kawaguchi, Y., and Kubota, Y. (1997). GABAergic cell subtypes and their synaptic connections in rat frontal cortex. Cereb. Cortex 7, 476-486.

Kessaris, N., Magno, L., Rubin, A. N., and Oliveira, M. G. (2014). Genetic programs controlling cortical interneuron fate. Curr. Opin. Neurobiol 26, 79-87. doi: 10.1016/j.conb.2013.12.012

Klausberger, T., and Somogyi, P. (2008). Neuronal diversity and temporal dynamics: the unity of hippocampal circuit operations. Science 321, 53-57. doi: $10.1126 /$ science. 1149381

Komada, M., Iguchi, T., Takeda, T., Ishibashi, M., and Sato, M. (2013). Smoothened controls cyclin D2 expression and regulates the generation of intermediate progenitors in the developing cortex. Neurosci. Lett. 547, 87-91. doi: 10.1016/j.neulet.2013.05.006

Lee, S., Hjerling-Leffler, J., Zagha, E., Fishell, G., and Rudy, B. (2010). The largest group of superficial neocortical gabaergic interneurons expresses ionotropic serotonin receptors. J. Neurosci. 30, 16796-16808. doi: 10.1523/jneurosci.186910.2010

Le Magueresse, C., and Monyer, H. (2013). GABAergic interneurons shape the functional maturation of the cortex. Neuron 77, 388-405. doi: 10.1016/j.neuron.2013.01.011

Levitt, P., Eagleson, K. L., and Powell, E. M. (2004). Regulation of neocortical interneuron development and the implications for neurodevelopmental disorders. Trends Neurosci. 27, 400-406. doi: 10.1016/j.tins.2004. 05.008

Li, G., Adesnik, H., Li, J., Long, J., Nicoll, R. A., Rubenstein, J. L., et al. (2008). Regional distribution of cortical interneurons and development of inhibitory tone are regulated by Cxcl12/Cxcr4 signaling. J. Neurosci. 28, 1085-1098. doi: 10.1523/JNEUROSCI.4602-07.2008

Lin, P. Y., Hinterneder, J. M., Rollor, S. R., and Birren, S. J. (2007). Non-cell-autonomous regulation of GABAergic neuron development by neurotrophins and the p75 receptor. J. Neurosci. 27, 12787-12796. doi: 10.1523/JNEUROSCI.3302-07.2007

Liodis, P., Denaxa, M., Grigoriou, M., Akufo-Addo, C., Yanagawa, Y., and Pachnis, V. (2007). Lhx6 activity is required for the normal migration and specification of cortical interneuron subtypes. J. Neurosci. 27, 3078-3089. doi: 10.1523/JNEUROSCI.3055-06.2007

Lodato, S., Rouaux, C., Quast, K. B., Jantrachotechatchawan, C., Studer, M., Hensch, T. K., et al. (2011). Excitatory projection neuron subtypes control the distribution of local inhibitory interneurons in the cerebral cortex. Neuron 69, 763-779. doi: 10.1016/j.neuron.2011. 01.015

Lopez-Bendito, G., Sanchez-Alcaniz, J. A., Pla, R., Borrell, V., Pico, E., Valdeolmillos, M., et al. (2008). Chemokine signaling controls intracortical migration and final distribution of GABAergic interneurons. J. Neurosci. 28, 1613-1624. doi: 10.1523/JNEUROSCI.4651-07.2008

Lukaszewicz, A., Savatier, P., Cortay, V., Kennedy, H., and Dehay, C. (2002). Contrasting effects of basic fibroblast growth factor and neurotrophin 3 on cell cycle kinetics of mouse cortical stem cells. J. Neurosci. 22, 6610-6622.

Ma, Y., Hu, H., Berrebi, A. S., Mathers, P. H., and Agmon, A. (2006). Distinct subtypes of somatostatin-containing neocortical interneurons revealed in transgenic mice. J. Neurosci. 26, 5069-5082. doi: 10.1523/JNEUROSCI.0661 06.2006

Magrassi, L., Ehrlich, M. E., Butti, G., Pezzotta, S., Govoni, S., and Cattaneo, E. (1998). Basal ganglia precursors found in aggregates following embryonic transplantation adopt a striatal phenotype in heterotopic locations. Development 125 , 2847-2855.

Markram, H., Toledo-Rodriguez, M., Wang, Y., Gupta, A., Silberberg, G., and $\mathrm{Wu}$, C. (2004). Interneurons of the neocortical inhibitory system. Nat. Rev. Neurosci. 5, 793-807. doi: 10.1038/nrn1519 
Massi, L., Lagler, M., Hartwich, K., Borhegyi, Z., Somogyi, P., and Klausberger, T. (2012). Temporal dynamics of parvalbumin-expressing axo-axonic and basket cells in the rat medial prefrontal cortex in vivo. J. Neurosci. 32, 16496-16502. doi: 10.1523/JNEUROSCI.3475-12.2012

McGarry, L. M., Packer, A. M., Fino, E., Nikolenko, V., Sippy, T., and Yuste, R. (2010). Quantitative classification of somatostatin-positive neocortical interneurons identifies three interneuron subtypes. Front. Neural. Cir. 4:12. doi: 10.3389/fncir.2010.00012

Miyoshi, G., Butt, S. J. B., Takebayashi, H., and Fishell, G. (2007). Physiologically distinct temporal cohorts of cortical interneurons arise from telencephalic Olig2-expressing precursors. J. Neurosci. 27, 7786-7798. doi: 10.1523/JNEUROSCI.1807-07.2007

Miyoshi, G., and Fishell, G. (2011). GABAergic interneuron lineages selectively sort into specific cortical layers during early postnatal development. Cereb. Cortex 21, 845-852. doi: 10.1093/cercor/bhq155

Miyoshi, G., Hjerling-Leffler, J., Karayannis, T., Sousa, V. H., Butt, S. J. B., Battiste, J., et al. (2010). Genetic fate mapping reveals that the caudal ganglionic eminence produces a large and diverse population of superficial cortical interneurons. J. Neurosci. 30, 1582-1594. doi: 10.1523/JNEUROSCI.451509.2010

Morales, M., and Bloom, F. E. (1997). The 5-HT3 receptor is present in different subpopulations of GABAergic neurons in the rat telencephalon. J. Neurosci. 17, 3157-3167.

Nery, S., Fishell, G., and Corbin, J. G. (2002). The caudal ganglionic eminence is a source of distinct cortical and subcortical cell populations. Nat. Neurosci. 5, 1279-1287. doi: 10.1038/nn971

Neves, G., Shah, M. M., Liodis, P., Achimastou, A., Denaxa, M., Roalfe, G., et al. (2013). The LIM homeodomain protein Lhx6 regulates maturation of interneurons and network excitability in the mammalian cortex. Cereb. Cortex 23, 1811-1823. doi: 10.1093/cercor/bhs159

Parnavelas, J. G., Barfield, J. A., Franke, E., and Luskin, M. B. (1991). Separate progenitor cells give rise to pyramidal and nonpyramidal neurons in the rat telencephalon. Cereb. Cortex 1, 463-468.

Parthasarathy, S., Srivatsa, S., Nityanandam, A., and Tarabykin, V. (2014). Ntf3 acts downstream of Sip1 in cortical postmitotic neurons to control progenitor cell fate through feedback signaling. Development 141, 3324-3330. doi: 10.1242/dev. 114173

Pesold, C., Liu, W. S., Guidotti, A., Costa, E., and Caruncho, H. J. (1999). Cortical bitufted, horizontal, and Martinotti cells preferentially express and secrete reelin into perineuronal nets, nonsynaptically modulating gene expression. Proc. Natl. Acad. Sci. U.S.A. 96, 3217-3222.

Pla, R., Borrell, V., Flames, N., and Marín, O. (2006). Layer acquisition by cortical GABAergic interneurons is independent of Reelin signaling. J. Neurosci. 26, 6924-6934. doi: 10.1523/JNEUROSCI.0245-06. 2006

Powell, E. M., Campbell, D. B., Stanwood, G. D., Davis, C., Noebels, J. L., and Levitt, P. (2003). Genetic disruption of cortical interneuron development causes region- and GABA cell type-specific deficits, epilepsy, and behavioral dysfunction. J. Neurosci. 23, 622-631.

Rakic, P. (1988). Specification of cerebral cortical areas. Science 241, 170-176.

Rakic, P. (2009). Evolution of the neocortex: a perspective from developmental biology. Nat. Rev. Neurosci. 10, 724-735. doi: 10.1038/ nrn2719

Ramamoorthi, K., and Lin, Y. (2011). The contribution of GABAergic dysfunction to neurodevelopmental disorders. Trends Mol. Med. 17, 452-462. doi: 10.1016/j.molmed.2011.03.003

Rosenberg, S. S., and Spitzer, N. C. (2011). Calcium signaling in neuronal development. Cold Spring Harb. Perspect. Biol. 3:a004259. doi: 10.1101/cshperspect.a004259

Rouaux, C., and Arlotta, P. (2010). Fezf2 directs the differentiation of corticofugal neurons from striatal progenitors in vivo. Nat. Neurosci. 13, 1345-1347. doi: 10.1038/nn.2658

Rudy, B., Fishell, G., Lee, S., and Hjerling-Leffler, J. (2010). Three groups of interneurons account for nearly $100 \%$ of neocortical GABAergic neurons. Dev. Neurobiol. 71, 45-61. doi: 10.1002/dneu.20853

Schuurmans, C., and Guillemot, F. (2002). Molecular mechanisms underlying cell fate specification in the developing telencephalon. Curr. Opin. Neurobiol. 12, 26-34.
Seuntjens, E., Nityanandam, A., Miquelajauregui, A., Debruyn, J., Stryjewska, A., Goebbels, S., et al. (2009). Sipl regulates sequential fate decisions by feedback signaling from postmitotic neurons to progenitors. Nat. Neurosci. 12, 1373-1380. doi: 10.1038/nn.2409

Somogyi, P., and Klausberger, T. (2005). Defined types of cortical interneurone structure space and spike timing in the hippocampus. J. Physiol. 562, 9-26. doi: 10.1113/jphysiol.2004.078915

Sousa, V. H., Miyoshi, G., Hjerling-Leffler, J., Karayannis, T., and Fishell, G. (2009). Characterization of Nkx6-2-derived neocortical interneuron lineages. Cereb. Cortex 19(Suppl. 1), i1-i10. doi: 10.1093/cercor/bhp038

Spitzer, N. C. (2012). Activity-dependent neurotransmitter respecification. Nat. Rev. Neurosci. 13, 94-106. doi: 10.1038/nrn3154

Steriade, M., Timofeev, I., Durmuller, N., and Grenier, F. (1998). Dynamic properties of corticothalamic neurons and local cortical interneurons generating fast rhythmic (30-40 Hz) spike bursts. J. Neurophysiol. 79, 483-490.

Stuhmer, T., Puelles, L., Ekker, M., and Rubenstein, J. L. (2002). Expression from a Dlx gene enhancer marks adult mouse cortical GABAergic neurons. Cereb. Cortex 12, 75-85.

Sussel, L., Marin, O., Kimura, S., and Rubenstein, J. L. (1999). Loss of Nkx2.1 homeobox gene function results in a ventral to dorsal molecular respecification within the basal telencephalon: evidence for a transformation of the pallidum into the striatum. Development 126, 3359-3370.

Taniguchi, H., Lu, J., and Huang, Z. J. (2013). The spatial and temporal origin of chandelier cells in mouse neocortex. Science 339, 70-74. doi: 10.1126/science. 1227622

Valcanis, H., and Tan, S.-S. (2003). Layer specification of transplanted interneurons in developing mouse neocortex. J. Neurosci. 23, 5113-5122.

Van Eden, C. G., Mrzljak, L., Voorn, P., and Uylings, H. B. (1989). Prenatal development of GABA-ergic neurons in the neocortex of the rat. J. Comp. Neurol. 289, 213-227. doi: 10.1002/cne.902890204

Vitalis, T., Ansorge, M. S., and Dayer, A. G. (2013). Serotonin homeostasis and serotonin receptors as actors of cortical construction: special attention to the 5-HT3A and 5-HT6 receptor subtypes. Front. Cell Neurosci. 7:93. doi: 10.3389/fncel.2013.00093

Vitalis, T., Cases, O., Passemard, S., Callebert, J., and Parnavelas, J. G. (2007). Embryonic depletion of serotonin affects cortical development. Eur. J. Neurosci. 26, 331-344. doi: 10.1111/j.1460-9568.2007.05661.x

Vogt, D., Hunt, R. F., Mandal, S., Sandberg, M., Silberberg, S. N., Nagasawa, T., et al. (2014). Lhx6 directly regulates Arx and CXCR7 to determine cortical interneuron fate and laminar position. Neuron 82, 350-364. doi: 10.1016/j.neuron.2014.02.030

Vucurovic, K., Gallopin, T., Ferezou, I., Rancillac, A., Chameau, P., Van Hooft, J. A., et al. (2010). Serotonin 3A receptor subtype as an early and protracted marker of cortical interneuron subpopulations. Cereb. Cortex 20, 2333-2347. doi: 10.1093/cercor/ bhp310

Wichterle, H., Turnbull, D. H., Nery, S., Fishell, G., and Alvarez-Buylla, A. (2001). In utero fate mapping reveals distinct migratory pathways and fates of neurons born in the mammalian basal forebrain. Development 128, 3759-3771.

Wonders, C. P., and Anderson, S. A. (2006). The origin and specification of cortical interneurons. Nat. Rev. Neurosci. 7, 687-696. doi: 10.1038/ nrn1954

Xu, Q., Cobos, I., Cruz, E. D. L., Rubenstein, J. L., Anderson, S. A., and De La Cruz, E. (2004). Origins of Cortical Interneuron Subtypes. J. Neurosci. 24, 2612-2622. doi: 10.1523/JNEUROSCI.5667-03.2004

Xu, Q., Guo, L., Moore, H., Waclaw, R. R., Campbell, K., and Anderson, S. A. (2010a). Sonic hedgehog signaling confers ventral telencephalic progenitors with distinct cortical interneuron fates. Neuron 65, 328-340. doi: 10.1016/j.neuron.2010. 01.004

Xu, X., Roby, K. D., and Callaway, E. M. (2010b). Immunochemical characterization of inhibitory mouse cortical neurons: three chemically distinct classes of inhibitory cells. J. Comp. Neurol. 518, 389-404. doi: 10.1002/cne.22229

Xu, Q., Wonders, C. P., and Anderson, S. A. (2005). Sonic hedgehog maintains the identity of cortical interneuron progenitors in the ventral telencephalon. Development 132, 4987-4998. doi: 10.1242/dev.02090

Zhao, Y., Flandin, P., Long, J. E., Cuesta, M. D., Westphal, H., and Rubenstein, J. L. (2008). Distinct molecular pathways for development of telencephalic 
interneuron subtypes revealed through analysis of Lhx6 mutants. J. Comp. Neurol. 510, 79-99. doi: 10.1002/cne.21772

Conflict of Interest Statement: The authors declare that the research was conducted in the absence of any commercial or financial relationships that could be construed as a potential conflict of interest.
Copyright $\odot 2015$ Brandão and Romcy-Pereira. This is an open-access article distributed under the terms of the Creative Commons Attribution License (CC BY). The use, distribution or reproduction in other forums is permitted, provided the original author(s) or licensor are credited and that the original publication in this journal is cited, in accordance with accepted academic practice. No use, distribution or reproduction is permitted which does not comply with these terms. 